\title{
Dose banding as an alternative to body surface area-based dosing of chemotherapeutic agents
}

\author{
E Chatelut*, , ML White-Koning', RHJ Mathijssen², F Puisset', SD Baker ${ }^{3}$ and A Sparreboom ${ }^{3}$ \\ 'EA 4553 Institut Claudius-Regaud, Université de Toulouse, 20-24 rue du Pont St Pierre, 31052 Toulouse Cedex, France; ${ }^{2}$ Erasmus University Medical \\ Center - Daniel den Hoed Cancer Center, Rotterdam, The Netherlands; ${ }^{3}$ St Jude Children's Research Hospital, Memphis, TN, USA
}

BACKGROUND: Dose banding is a recently suggested dosing method that uses predefined ranges (bands) of body surface area (BSA) to calculate each patient's dose by using a single BSA-value per band. Thus, drugs with sufficient long-term stability can be prepared in advance. The main advantages of dose banding are to reduce patient waiting time and improve pharmacy capacity planning; additional benefits include reduced medication errors, reduced drug wastage, and prospective quality control. This study compares dose banding with individual BSA dosing and fixed dose according to pharmacokinetic criteria.

METHODS: Three BSA bands were defined: BSA $<1.7 \mathrm{~m}^{2}, 1.7 \mathrm{~m}^{2} \leqslant \mathrm{BSA}<1.9 \mathrm{~m}^{2}, \mathrm{BSA} \geqslant 1.9 \mathrm{~m}^{2}$ and each patient dose was calculated based on a unique BSA-value per band $\left(1.55,1.80\right.$, and $2.05 \mathrm{~m}^{2}$, respectively). By using individual clearance values of six drugs (cisplatin, docetaxel, paclitaxel, doxorubicin, irinotecan, and topotecan) from 1012 adult cancer patients in total, the AUCs corresponding to three dosing methods (BSA dosing, dose banding, and fixed dose) were compared with a target AUC for each drug.

RESULTS: For all six drugs, the per cent variation in individual dose obtained with dose banding compared with BSA dosing ranged between $-14 \%$ and $+22 \%$, and distribution of AUC values was very similar with both dosing methods. In terms of reaching the target AUC, there was no significant difference in precision between dose banding and BSA dosing, except for paclitaxel (32.0\% vs $30.7 \%$, respectively; $P<0.05)$. However, precision was significantly better for BSA dosing compared with fixed dose for four out of six drugs.

CONCLUSION: For the studied drugs, implementation of dose banding should be considered as it entails no significant increase in interindividual plasma exposure.

British Journal of Cancer (2012) I 07, I I00-1 |06. doi:I0.1038/bjc.20 I2.357 www.bjcancer.com

Published online 28 August 2012

(c) 2012 Cancer Research UK

Keywords: dose banding; dose standardisation; anticancer drugs; fixed dose; chemotherapy

Drug dosing is a key issue in oncology due to the 'dose-effect' relationship of cytotoxics. The ideal (recommended) dose for a specific patient is the highest dose associated with an acceptable toxicity. This dose is known as the maximum tolerated dose determined during the phase I trial. The current practice of using body surface area (BSA) in dosing anticancer drugs was implemented in clinical oncology half a century ago by Pinkel (1958). Based on the theory that large patients have a higher elimination capacity, it is assumed that those patients need to be given higher doses than smaller patients to reach equal drug concentrations. Several studies have shown that both toxicity and efficacy of cytotoxics are related to plasma drug exposure (i.e., area under the curve of the plasma drug concentrations vs time, AUC; Gurney, 1996). Most of these studies and some others (Sparreboom, 2005) have shown that the only pharmacokinetic (PK) parameter significantly associated with individual AUC, elimination clearance (CL), is either poorly or not correlated with BSA for most cytotoxics (Reilly and Workman, 1993; Mathijssen et al, 2007). As a consequence of this poor or absent correlation,

*Correspondence: Dr E Chatelut;

E-mail: chatelut.etienne@claudiusregaud.fr

Received 8 May 2012; revised 27 June 2012; accepted 17 July 2012; published online 28 August 2012 the current practice based on BSA dosing is associated with a large interindividual variability in terms of toxicity, largely due to heterogeneous plasma drug exposure between patients treated by the same chemotherapy protocol. Unfortunately, for many anticancer drugs currently under use, little is known concerning the characteristics that influence clearance (and consequently are likely to affect individual AUC), and thus no satisfactory alternatives to BSA dosing have been suggested that could improve the efficacy of these drugs.

Dose banding has recently been suggested to optimise chemotherapy preparation (Plumridge and Sewell, 2001; Pouliquen et al, 2011). Ranges (or bands) of BSA, and corresponding mid-points of each band are predefined. The individual dose of a particular patient is calculated according to a single BSA-value per band, usually the mid-point of the BSA band in which the actual BSA of the patient lies. Thanks to this system chemotherapy provision can be rationalised and chemotherapies can be prepared in advance for drugs with sufficient long-term drug stability. The main advantages of dose banding are to reduce patient waiting time and to improve capacity planning of the pharmacy production, but additional benefits can also be found, such as reduced potential for medication errors, reduced drug wastage, and prospective quality control of preparations. While the use of dose banding is unlikely to improve efficacy, it is 
of primary importance to evaluate whether it can be shown to be no worse than BSA dosing because of all the above-mentioned advantages of this method.

The objective of the present analysis was to compare BSA dosing (as the current practice), dose banding, and fixed dose according to PK criteria. By using individual values of clearance of six drugs (cisplatin, docetaxel, paclitaxel, doxorubicin, irinotecan, and topotecan) from a total of 1012 adult cancer patients, the AUCs corresponding to each of these three dosing methods were compared with a target value of AUC for each drug.

\section{PATIENTS AND METHODS}

\section{Data collection}

The study drugs included cisplatin $(n=283)$, docetaxel $(n=169)$, doxorubicin $(n=103)$, irinotecan $(n=187)$, paclitaxel $(n=80)$, and topotecan $(n=190)$. These agents were considered because of the known differences in their primary pathways of elimination. All patients were at least 18 years old and provided written informed consent for enrolment on studies approved by the local review boards. Additional common eligibility criteria included: (a) life expectancy of at least 12 weeks; (b) performance status of $0-2$; (c) no chemotherapy, hormonal therapy, or radiotherapy for at least 4 weeks before enrolment; (d) adequate contraception and a negative pregnancy test result for women of childbearing age; and (e) adequate bone marrow function, renal function, and hepatic function (i.e., patients with severe renal or hepatic impairment were excluded). Simultaneous use of any medication, dietary supplements, or other compounds known to inhibit, induce, or otherwise affect the pharmacokinetics of the study drugs was not allowed.

\section{Sample collection and PK analysis}

Preliminary PK data obtained from (subsets of) the studied patient populations have been published previously for cisplatin (de Jongh et al, 2001), docetaxel (Rudek et al, 2004), doxorubicin (Rudek et al, 2004), irinotecan (de Jong et al, 2004), paclitaxel (Henningsson et al, 2005), and topotecan (Leger et al, 2004).

Cisplatin Patients were treated with cisplatin monotherapy or cisplatin-based combination therapy with oral etoposide, irinotecan, oral topotecan, or docetaxel. The drug was administered as a 3 -h infusion at doses ranging from 50 to $100 \mathrm{mg} \mathrm{m}^{-2}$, with treatment cycles repeated every week or every 3 weeks. Blood samples were obtained immediately before infusion, at 1 and $2 \mathrm{~h}$ after the start of the infusion, at the end of infusion, and at $0.5,1,2$, 3 , and $18 \mathrm{~h}$ after the end of infusion. Plasma concentrations of unbound cisplatin were determined by atomic absorption spectrometry (de Jongh et al, 2001).

Docetaxel Patients were treated with docetaxel alone or combined with capecitabine, cisplatin, doxorubicin with or without marimastat, or methotrexate. Docetaxel was administered as a 1-h infusion at doses ranging from 55 to $100 \mathrm{mg} \mathrm{m}^{-2}$. Blood samples were obtained immediately before docetaxel administration; at 30 min after the start of infusion, at the end of infusion, and at 1, 3, 5,23 , and $48 \mathrm{~h}$ after the end of infusion. Determination of docetaxel was performed using high-performance liquid chromatography (HPLC) with UV detection (Rudek et al, 2004).

Doxorubicin Patients were treated with doxorubicin monotherapy or with doxorubicin-based combination therapy with cyclophosphamide, docetaxel, or paclitaxel. Doxorubicin was administered as an intravenous bolus $(5 \mathrm{~min})$, a short infusion (15-20 min), or as a 1- to 3-h infusion at doses ranging from 40 to
$75 \mathrm{mg} \mathrm{m}^{-2}$. Blood samples were taken immediately before infusion, directly after infusion, and $30 \mathrm{~min}$ and 1, 2, 4, 7, 12, 24, and $48 \mathrm{~h}$ post infusion. Concentrations of doxorubicin in plasma were determined by HPLC with fluorescence detection (Rudek et al, 2004).

Irinotecan Irinotecan was administered either alone or in combination with cisplatin as a 90 -min continuous intravenous infusion at a dose level between 175 and $350 \mathrm{mg} \mathrm{m}^{-2}$ or at a flat dose of $600 \mathrm{mg}$. Blood samples were collected before infusion, at 0.5 and $1.5 \mathrm{~h}$ during infusion, and 10, 20, and $30 \mathrm{~min}$ and $1,2,3$, $3.5,4,5,6.5,10.5,24,30.5,48$, and $54.5 \mathrm{~h}$ after the end of infusion. Concentrations of irinotecan and its active metabolite SN-38 were determined by HPLC with fluorescence detection (de Jong et al, 2004).

Paclitaxel Paclitaxel was supplied as a concentrated solution in cremophor-ethanol. The drug was administered as a single agent or in combination with carboplatin. Paclitaxel was administered intravenously over 1,3 , or $24 \mathrm{~h}$ at doses ranging from 50 to $225 \mathrm{mg} \mathrm{m}^{-2}$. Blood samples were obtained at the following time points: immediately before infusion, and $0.5,1,1.5,2,2.5,3,5,7$, $13,25,33$, and $49 \mathrm{~h}$ after the start of paclitaxel infusion (1-h schedule); 1, 2, 3, 3.08, 3.25, 3.5, 4, 4.5, 5, 7, 9, 15, 21, 27, 35, and $51 \mathrm{~h}$ after the start of infusion (3-h schedule); or at 1, 22, 23, 23.92, $24.08,24.15,25,26,27,30,36$, and $45 \mathrm{~h}$ after the start of infusion (24-h schedule). Determination of total paclitaxel was performed by HPLC with UV detection (Henningsson et al, 2005).

Topotecan Topotecan was administered to patients once daily for 5 consecutive days every 3 weeks (dose, $0.2-2.4 \mathrm{mg} \mathrm{m}^{-2}$ or a fixed dose of $4 \mathrm{mg}$ ), once daily for $7,10,13$, or 21 days (dose, $0.20-1.00 \mathrm{mg} \mathrm{m}^{-2}$ ), or twice daily for 5,10 , or 21 days (dose, $0.15-2.70 \mathrm{mg} \mathrm{m}^{-2}$ ). The drug was administered as a single agent or in combination with cisplatin. Sample collection was performed before dosing, at $15 \mathrm{~min}$ after the start of infusion, immediately before the end of infusion, and at 15 and $30 \mathrm{~min}$ and 1, 2, 3, 4, 6, 8, and $10 \mathrm{~h}$ after the end of infusion. Determination of topotecan was performed by HPLC with fluorescence detection (Leger et al, 2004).

Clearance, volume of distribution, and terminal half-life for each drug were derived from individual plasma concentration-time profiles using non-compartmental analysis with the software package WinNonlin (Scientific Consultant, Apex, NC, USA) Professional Version 5.0 (Pharsight Corporation, Mountain View, CA, USA), model 202 (plasma data, constant infusion).

For each drug, the target AUC was calculated by using the mean value of observed CL expressed in $\mathrm{Lh}^{-1} \mathrm{~m}^{-2}$, and the dose $\left(\mathrm{mg} \mathrm{m}^{-2}\right)$ corresponding to a widely used standard protocol: target AUC $=$ standard dose $\left(\mathrm{mg} \mathrm{m}^{-2}\right) /$ mean observed CL $\left(\mathrm{L} \mathrm{h}^{-1} \mathrm{~m}^{-2}\right)$.

Body surface area was calculated using Dubois' formula: BSA $\left(\mathrm{m}^{2}\right)=0.007184 \times$ height $(\mathrm{cm})^{0.725} \times$ weight $(\mathrm{kg})^{0.425}$. Three doses were calculated for each patient: DoseBSA corresponding to standard dose $\left(\mathrm{mg} \mathrm{m}^{-2}\right)$ multiplied by individual BSA; DoseFIXED corresponding to standard dose $\left(\mathrm{mg} \mathrm{m}^{-2}\right)$ multiplied by mean BSA; DoseBAND corresponding to standard dose $\left(\mathrm{mg} \mathrm{m}^{-2}\right)$ multiplied by relevant BSA band value: respectively $1.55,1.80$, or $2.05 \mathrm{~m}^{2}$ depending on which of the following three BSA bands: BSA $<1.7 \mathrm{~m}^{2}, 1.7 \mathrm{~m}^{2} \leqslant \mathrm{BSA}<1.9 \mathrm{~m}^{2}$, or BSA $\geqslant 1.9 \mathrm{~m}^{2}$ the BSA of the patient belongs to. Cutoff values for the BSA bands were inspired from a previous dose-banding methodology used by Pouliquen et al (2011).

The relative difference between each dose calculation method and DoseBSA was calculated in the following way: relative difference $=(($ DoseStudied - DoseBSA $) /$ DoseBSA $) \times 100 \quad($ where DoseStudied is DoseFIXED or DoseBAND). The absolute value of relative difference $=(\mid$ DoseStudied - DoseBSA $\mid /$ DoseBSA $) \times 100$ 
was also calculated. Mean and standard deviation (s.d.) for these quantities were given as well as minimum and maximum values for relative difference. As an indication of dose modification, the percentage of values where absolute value of relative difference was $>10 \%$ was also given.

For each patient, the AUC corresponding to each of these three dose determination methods was calculated: Dose (mg)/actual patient $\mathrm{CL}\left(\mathrm{Lh}^{-1}\right)$ (e.g., AUCBSA = DoseBSA/CL); the percentage error of AUC (pi) corresponding to each dose was calculated: for

Table I Patient $(n=10 \mid 2)$ and drug characteristics for each patient group

\begin{tabular}{|c|c|c|c|c|}
\hline Drug & $\begin{array}{c}\text { Number } \\
\text { of } \\
\text { patients }\end{array}$ & $\begin{array}{l}\text { BSA }\left(\mathrm{m}^{2}\right) \\
\text { Mean (s.d.) } \\
(\mathrm{min}-\mathrm{max})\end{array}$ & $\begin{array}{c}\text { Clearance } \\
\left(\mathrm{Lh}^{-1}\right) \\
\text { Mean } \\
\text { (CV\%) }\end{array}$ & $\begin{array}{c}\text { Clearance } \\
\left(\mathrm{Lh} \mathrm{h}^{-1} \mathrm{~m}^{-2}\right) \\
\text { Mean } \\
(\mathrm{CV} \%)\end{array}$ \\
\hline Cisplatin & 283 & $1.85(0.20)(1.29-2.39)$ & $56.2(22.2)$ & $30.3(19.6)$ \\
\hline Docetaxel & 169 & $1.85(0.20)(1.29-2.33)$ & $41.7(36.1)$ & $22.6(34.9)$ \\
\hline Paclitaxel & 80 & $1.78(0.21)(1.32-2.38)$ & $337.1(26.0)$ & $189.8(24.0)$ \\
\hline Doxorubicin & 103 & $1.81(0.21)(1.27-2.35)$ & $63.8(35.9)$ & $35.4(35.2)$ \\
\hline Topotecan & 190 & $1.79(0.22)(1.36-2.39)$ & $22.3(29.0)$ & $12.5(28.0)$ \\
\hline $\begin{array}{l}\text { Irinotecan } \\
\text { (SN-38) }\end{array}$ & 187 & $1.86(0.21)(1.32-2.36)$ & $649.5(82.9)$ & $347.4(83.0)$ \\
\hline $\begin{array}{l}\text { Irinotecan } \\
\text { (CPT II) }\end{array}$ & 187 & $1.86(0.21)(1.32-2.36)$ & $31.8(30.6)$ & $17.2(31.4)$ \\
\hline
\end{tabular}

Abbreviations: $\mathrm{BSA}=$ body surface area; $\mathrm{CV}=$ coefficient of variation. example, (AUCBSA - AUCtarget) $\times 100 /$ AUCtarget. The absolute value of relative difference $=(\mid$ AUCStudied - AUCtarget $\mid /$ AUCtarget) $\times 100$ was also calculated (where AUCstudied is AUCBSA, AUCFIXED, or AUCBAND). The precision (root mean square error, RMSE) corresponding to each dosing method was calculated in the following way: $\operatorname{RMSE}(\%)=\sqrt{\sum_{i=1}^{n}(\mathrm{pi})^{2} / n}$, where $n$ is the number of patients in each drug group.

The potential impact of using dose banding (DoseBAND) or fixed dose (DoseFIXED) was evaluated in three different ways. First, we calculated the relative difference and absolute value of the relative difference (described above) between DoseBAND or DoseFIXED on the one hand, and the reference DoseBSA on the other. Second, the absolute value of the relative difference in AUCs between DoseBAND or DoseFIXED and Dose BSA was compared using paired Student's $t$-tests. Finally, paired Student's $t$-tests were used to compare the precision (RMSE) of the AUCs obtained using DoseBAND or DoseFIXED with the precision of the AUC obtained with DoseBSA. For irinotecan, evaluation was performed using both irinotecan and SN-38 AUC.

\section{RESULTS}

A total of 1012 adult cancer patients were studied. Their BSA together with their mean PK parameters are detailed in Table 1. For all six drugs, the per cent change in individual dose compared with BSA dosing ranged between $-14 \%$ and $+22 \%$ for dose banding, and $-25 \%$ and $+44 \%$ for fixed dose (Table 2 ). In terms

Table 2 Percentage dose variation of fixed dose method (DoseFIXED) and dose-banding method (DoseBAND) relative to dose based on individual BSA (DoseBSA)

\begin{tabular}{|c|c|c|c|c|c|c|c|c|c|c|c|}
\hline & \multirow[b]{3}{*}{ Usual dose per $\mathrm{m}^{2}(\mathrm{mg})$} & \multicolumn{5}{|c|}{ DoseFIXED } & \multicolumn{5}{|c|}{ DoseBAND } \\
\hline & & \multicolumn{2}{|c|}{$\begin{array}{l}\text { Absolute value of } \\
\text { relative difference }\end{array}$} & \multicolumn{3}{|c|}{ Relative difference } & \multicolumn{2}{|c|}{$\begin{array}{l}\text { Absolute value of } \\
\text { relative difference }\end{array}$} & \multicolumn{3}{|c|}{ Relative difference } \\
\hline & & Mean (s.d.) & $\geqslant 10 \% a$ & Mean (s.d.) & Min & $\operatorname{Max}$ & Mean (s.d.) & $\geqslant 10 \%$ & Mean (s.d.) & Min & Max \\
\hline Cisplatin & 80 & $8.9(6.7)$ & 37 & $1.2(11.1)$ & -23 & +44 & $3.9(2.7)$ & 2 & $-0.3(4.8)$ & -14 & +20 \\
\hline Docetaxel & 100 & $8.9(7.0)$ & 35 & $1.2(11.3)$ & -21 & +43 & $4.1(2.9)$ & 3 & $0.01(5.1)$ & -12 & +20 \\
\hline Paclitaxel & 100 & $10.0(7.6)$ & 41 & $1.5(12.5)$ & -25 & +35 & $3.9(3.2)$ & 4 & $1.4(4.9)$ & -14 & +17 \\
\hline Doxorubicin & 60 & $9.2(7.3)$ & 41 & $1.3(11.7)$ & -23 & +42 & $4.1(3.2)$ & 4 & $0.003(5.2)$ & -13 & +22 \\
\hline Topotecan & 1.5 & 10.1 (6.8) & 43 & $1.5(12.2)$ & -25 & +31 & $4.4(2.9)$ & 4 & $-0.4(5.3)$ & -14 & +14 \\
\hline Irinotecan & 350 & $9.3(7.3)$ & 40 & $1.3(11.7)$ & -21 & +41 & $4.1(2.9)$ & 5 & $-0.9(5.0)$ & -13 & +17 \\
\hline
\end{tabular}

Relative difference $=(($ DoseStudied - DoseBSA $) /$ DoseBSA $) \times 100$ where DoseStudied is DoseFIXED or DoseBAND. Absolute value of relative difference $=(\mid$ DoseStudied DoseBSA//DoseBSA) $\times 100$. a Percentage of values where absolute value of relative difference is $>10 \%$.

Table 3 Differences in plasma exposure (AUC) using each dosing method (DoseBSA, DoseFIXED and DoseBAND) compared with target AUC (usual dose per $\mathrm{m}^{2} /$ mean $\mathrm{CL}, \mathrm{Lh}^{-1} \mathrm{~m}^{-2}$ )

\begin{tabular}{|c|c|c|c|c|c|c|}
\hline \multirow[b]{3}{*}{ Drug } & \multirow{2}{*}{\multicolumn{2}{|c|}{$\begin{array}{c}\text { AUC with DoseBSA } \\
\text { Absolute value } \\
\text { of relative difference }\end{array}$}} & \multirow{2}{*}{\multicolumn{2}{|c|}{$\begin{array}{c}\text { AUC with DoseBAND } \\
\text { Absolute value of } \\
\text { relative difference }\end{array}$}} & \multirow{2}{*}{\multicolumn{2}{|c|}{$\begin{array}{c}\text { AUC with DoseFIXED } \\
\text { Absolute value of } \\
\text { relative difference }\end{array}$}} \\
\hline & & & & & & \\
\hline & Mean (s.d.) & RMSE & Mean (s.d.) & RMSE & Mean (s.d.) & RMSE \\
\hline Cisplatin & $15.3(13.7)$ & 20.5 & $15.7(13.8)$ & 21.0 & $16.9 *(15.8)$ & $23.1 *$ \\
\hline Docetaxel & $32.8(32.6)$ & 46.2 & $33.3(33.1)$ & 46.9 & $35.8 *(36.2)$ & $50.8^{*}$ \\
\hline Paclitaxel & $21.8(21.7)$ & 30.7 & $23.2 *(22.2)$ & $32.0 *$ & $23.6(25.7)$ & $34.8 *$ \\
\hline Doxorubicin & $34.6(41.2)$ & 53.6 & $34.4(40.3)$ & 52.9 & $35.7(42.0)$ & 55.0 \\
\hline Topotecan & $22.3(20.8)$ & 30.4 & $23.0(20.9)$ & 31.1 & $23.8(25.0)$ & $34.5^{*}$ \\
\hline Irinotecan (SN-38) & $82.0(90.7)$ & 122.0 & $81.0(89.4)$ & 120.5 & $84.7(96.0)$ & 127.8 \\
\hline Irinotecan (CPTII) & $28.4(28.9)$ & 40.5 & $28.5(27.8)$ & 39.8 & $29.2(31.0)$ & 42.5 \\
\hline
\end{tabular}

Abbreviations: $\mathrm{AUC}=$ area under the curve; $\mathrm{BSA}=$ body surface area; $\mathrm{CL}=$ clearance; $\mathrm{RMSE}=$ root mean square error. $*$ Statistically significant difference $(P<0.05)$ between value and corresponding value with DoseBSA method. 

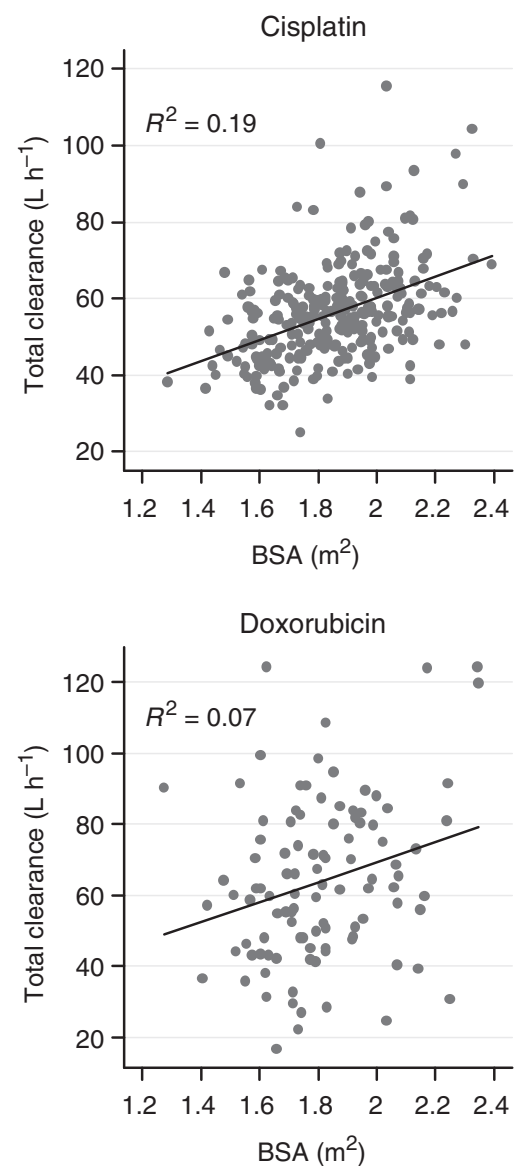

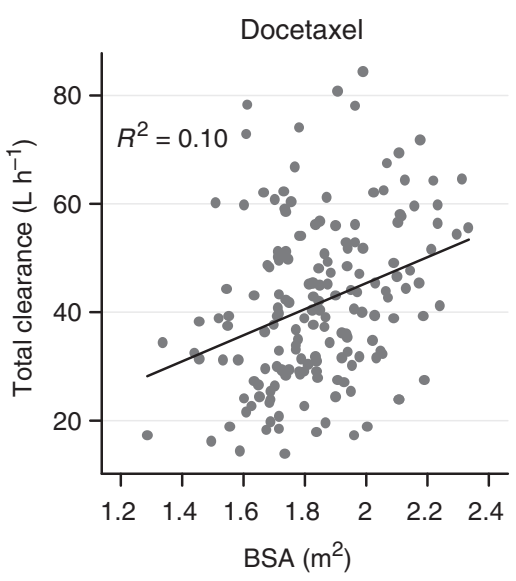

Topotecan

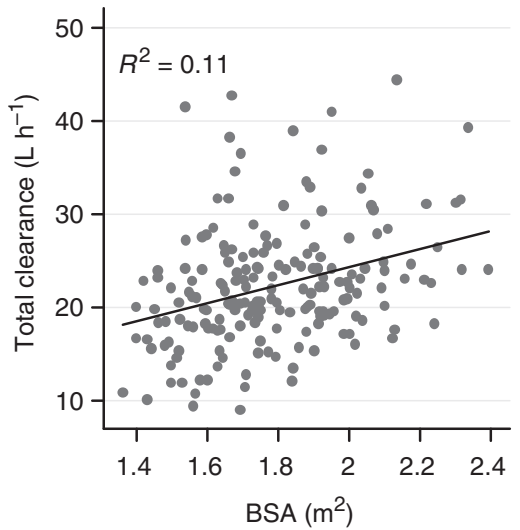

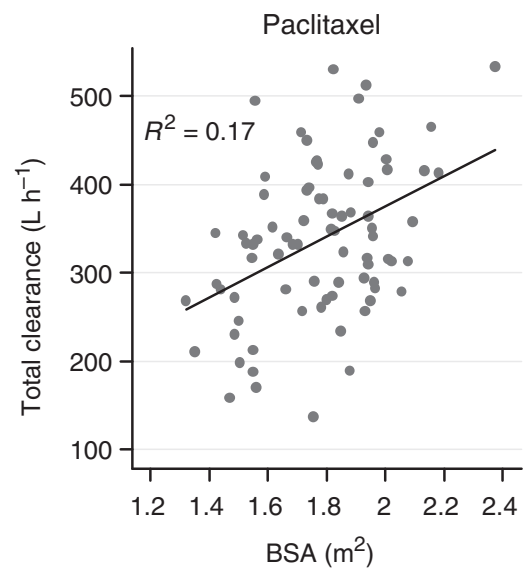

Irinotecan (SN-38)

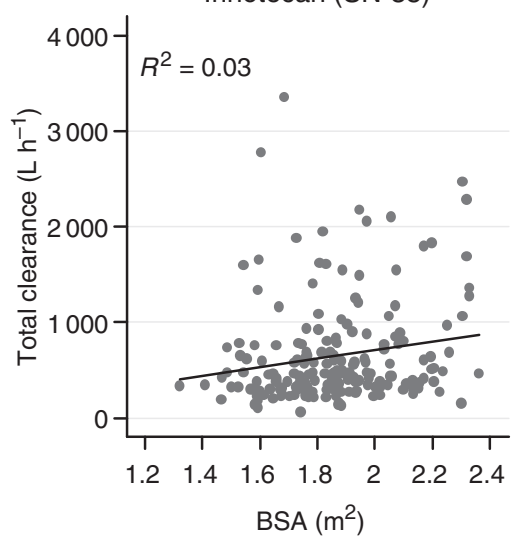

Figure I Relationship between body surface area (BSA) and drug clearance for all six drugs (for irinotecan, apparent clearance corresponding to SN-38 was considered). $R^{2}$ values indicate the contribution of BSA to the interindividual variability in clearance.

of capacity to attain the target AUC, precision was statistically significantly better for BSA dosing compared with fixed dose for four out of six drugs: cisplatin (20.5\% vs $23.1 \%$, respectively), docetaxel $(46.2 \%$ vs $50.8 \%)$, paclitaxel $(30.7 \%$ vs $34.8 \%)$, and topotecan $(30.4 \%$ vs $34.5 \%)$. For the remaining two drugs, there was no statistically significant difference in precision (doxorubicin: $53.6 \%$ vs 55.0\%; irinotecan (SN-38): $122.0 \%$ vs 127.8\%). However, the precision of dose banding was not significantly worse $(P<0.05)$ than BSA dosing, except paclitaxel $(32.0 \%$ vs $30.7 \%$, respectively) (Table 3 ). The precision values were very different for the six drugs ranging from $20 \%$ to $23 \%$ (according to dosing method) for cisplatin to $120-128 \%$ for SN-38, the active metabolite of irinotecan. However, for a specific drug the precision values were very close to each other, regardless of the dosing method used, which is a consequence of the very similar interindividual variability in clearance expressed in $\mathrm{L} \mathrm{h}^{-1} \mathrm{~m}^{-2}$ compared with values expressed in $\mathrm{Lh}^{-1}$ (Table 1).

\section{DISCUSSION}

In oncology as in every area of medicine, change of practice requires formal evaluation and validation. Current dosing practice for cytotoxics is based on BSA since chemotherapy protocols are evaluated according to this method during phase I to phase III trials. Although clearance is poorly correlated with BSA (Figure 1), this practice is partially validated by the precisions observed for BSA dosing and fixed dose, respectively (Mathijssen et al, 2007). Indeed, even though the precision values were very similar for BSA dosing and dose banding, those corresponding to BSA dosing were statistically significantly lower than those of fixed doses for cisplatin, docetaxel, paclitaxel, and topotecan. On the contrary, for all drugs but paclitaxel, dose banding was associated with a precision that was not significantly worse than that of BSA dosing. Although it was significantly different for paclitaxel, precision of dose banding (i.e., 32.0\%) was clinically similar to that of BSA dosing (i.e., 30.7\%). The distribution of the per cent error (Figures 2 and 3 ) confirms that dose banding is similar to BSA dosing, even for paclitaxel.

Dose banding was first mentioned in 1996 in a brief news article describing the system developed at the University Hospital Birmingham National Health Science Trust. It was suggested to rationalise chemotherapy provision. In particular, dose banding could represent an economic interest for chemotherapy compounding units. Indeed, BSA dosing implies individual preparation of doses for each patient. If chemotherapy is not administered (mainly in case of change in dose, change in treatment, or cancellation of treatment), then good manufacturing practices require that unused doses be thrown away. The cost of unused doses in case of change or cancellation of treatment could represent about $1 \%$ of a hospital cancer drug budget (Berhoune et al, 2011). Even if some changes in treatment could be prevented, the cost of unused treatment due to last minute changes in dose could not be reduced. With a dose-banding system, pharmacists would prepare doses not for a specific patient, so in case of cancellation of a planned dose for a patient, the prepared dose would not be wasted but used for another patient.

In 2001, Plumridge and Sewell defined dose banding as 'a system whereby, through agreement between prescribers and pharmacists, doses of intravenous cytotoxic drugs, calculated on an 

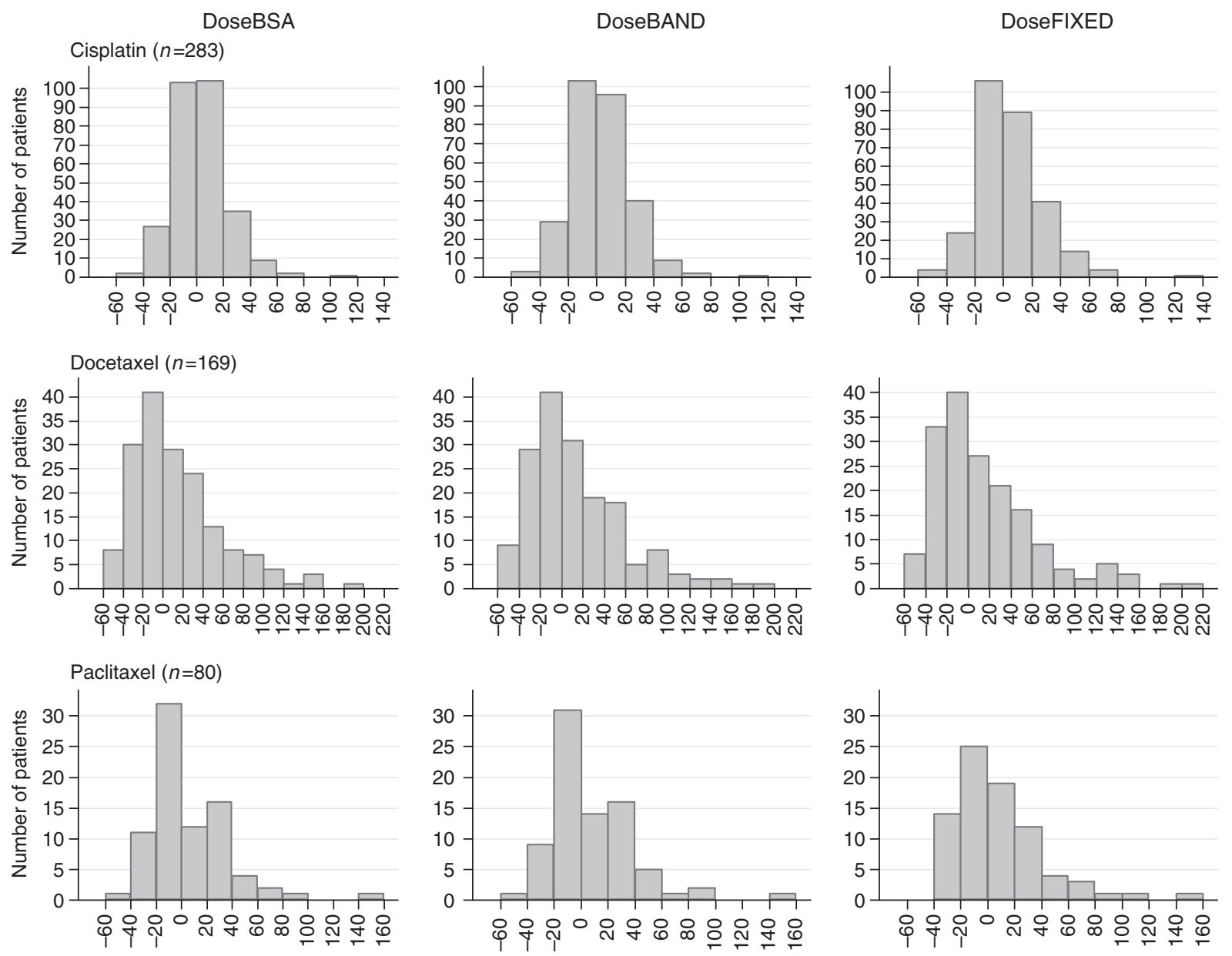

Figure 2 Frequency of percentage errors between individual AUC and target AUC using BSA dosing, dose banding, or fixed dose for cisplatin, docetaxel, and paclitaxel.

individualised basis, which are within defined ranges or bands, are approximated to predetermined standard doses; the maximum variation of the adjustment between the standard dose and the doses constituting each band is 5\% or less' (Plumridge and Sewell, 2001). The choice we made to use only three bands covering the total range of observed patients' BSA $\left(1.29-2.39 \mathrm{~m}^{2}\right)$ was associated with a wider range of variation between the doses corresponding to dose banding compared with those obtained using BSA dosing: from $-14 \%$ to $+22 \%$ when considering all six drugs. The major benefits of this strategy of limiting to three standardised doses would be to facilitate the planning of pharmacy production, and to reduce the number of infusion wastages (preprepared chemotherapies that are not used because no patient has a BSA corresponding to that particular band). A similar analysis was performed using five bands (rather than the three bands) with cutoff values of 1.525 , $1.725,1.875$, and $2.025 \mathrm{~m}^{2}$ (and corresponding values used for dose calculation of $1.50,1.65,1.80,1.95$, and $2.10 \mathrm{~m}^{2}$ ). As expected the precision in terms of achieving target AUC was not significantly different (data not shown). However, the benefit of this 5-band design was to decrease the proportion of patients with dose differing by $>10 \%$ compared with individual BSA dosing $(2-3 \%$ for the 5-band method vs 2-5\% for the 3-band method depending on the drug). Hence, this 5-band system may be considered as both more conservative and less contestable compared with the current BSA dosing practice. However, it also limits the benefit in terms of chemotherapy manufacturing within the hospital pharmacy department since it exposes to more unused (wasted) chemotherapy preparation. Another previously suggested criterion that could be considered when fixing the standardised doses would be to have the volumes to be taken from the commercial vials corresponding to that of the vials of the commercial drug. If overall the precision of dose banding is similar to that of BSA dosing, then the change in terms of plasma AUC may be as great as the change in dose (i.e., $-14 \%$ and $+22 \%$ by considering our database and the chosen dose-banding method) for a specific patient. The risk of observing high toxicity in a patient treated using a dose $22 \%$ higher than that which the patient would have received with BSA dosing, may be a limiting factor for the practice of dose banding. Indeed, one could not be certain that the $22 \%$ higher dose had no contribution to this high toxicity. However, high toxicities of cytotoxic treatment are observed daily due to the fact that BSA dosing can be responsible for overexposure in patients. This begs the question as to whether we should be more demanding for dose banding than we are for BSA dosing. Unfortunately, toxicity and efficacy data were not available in this study, which is why PK variables were used as a surrogate for such clinical endpoints. We evaluated only six cytotoxics but they present diverse PK properties. Indeed, renal clearance represents a substantial contribution to topotecan elimination, and cisplatin disposition is dominated by near covalent binding of the drug to serum proteins. The four other drugs are eliminated mainly by metabolism. Indeed, Taxanes are metabolised by the cytochrome P450 isozymes CYP3A4 (docetaxel) and CYP2C8 and CYP3A4 (paclitaxel), doxorubicin by aldoketoreductase, and SN-38, the active metabolite of irinotecan, by carboxylesterases and UDP glucuronosyltransferases, including UGT1A1 (Mathijssen et al, 2001).

For SN-38, the poor precision observed whatever the method of dose calculation used suggests that it may be relevant to explore 


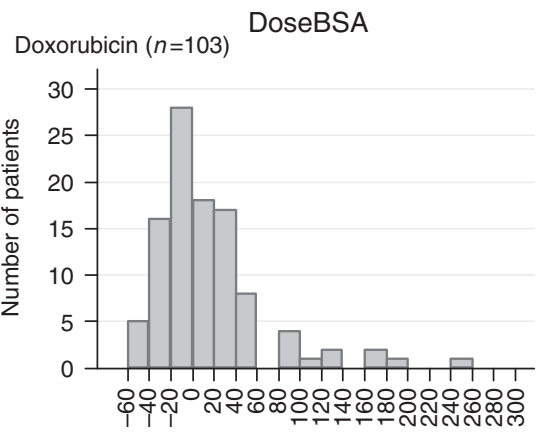

DoseBAND
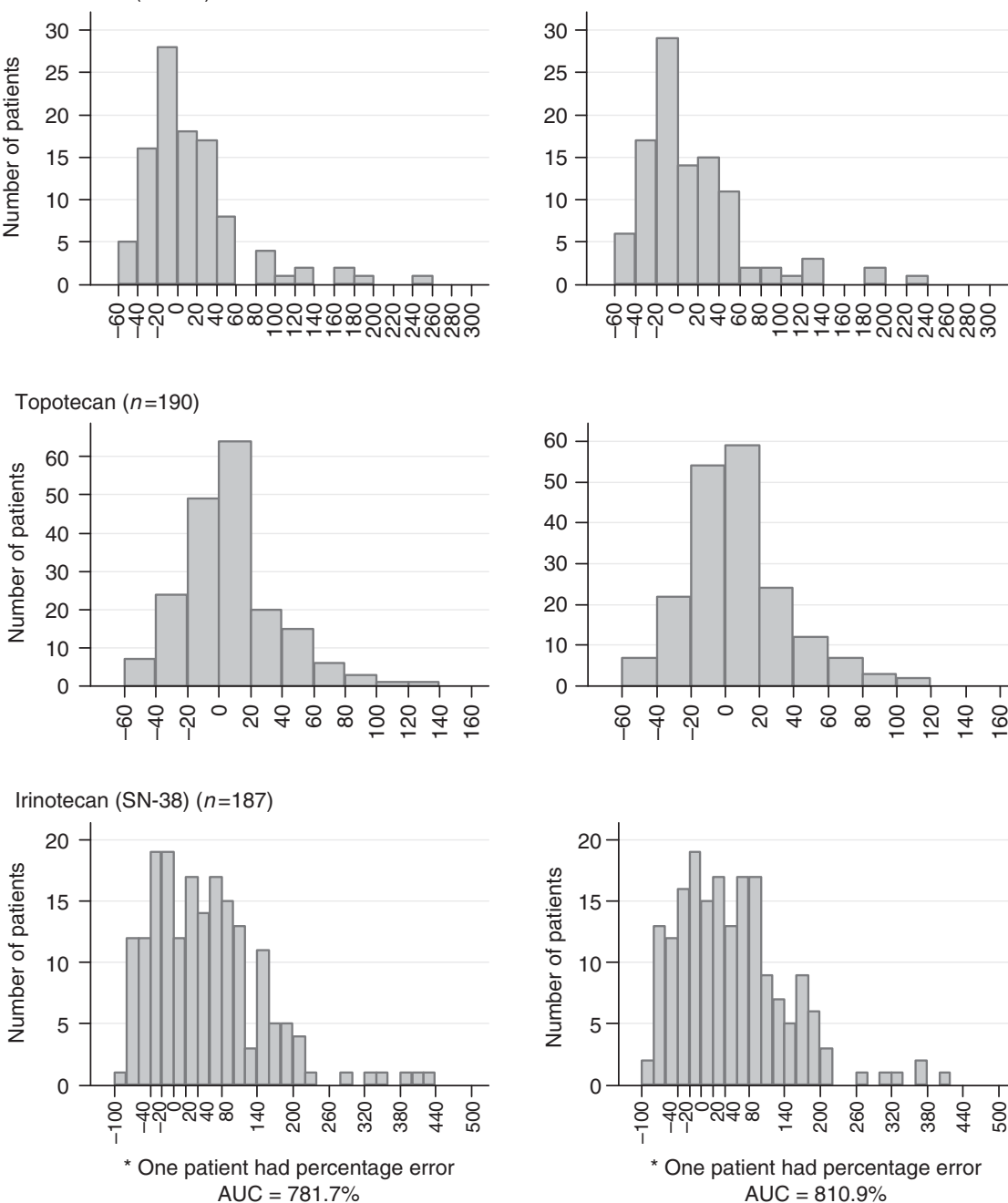
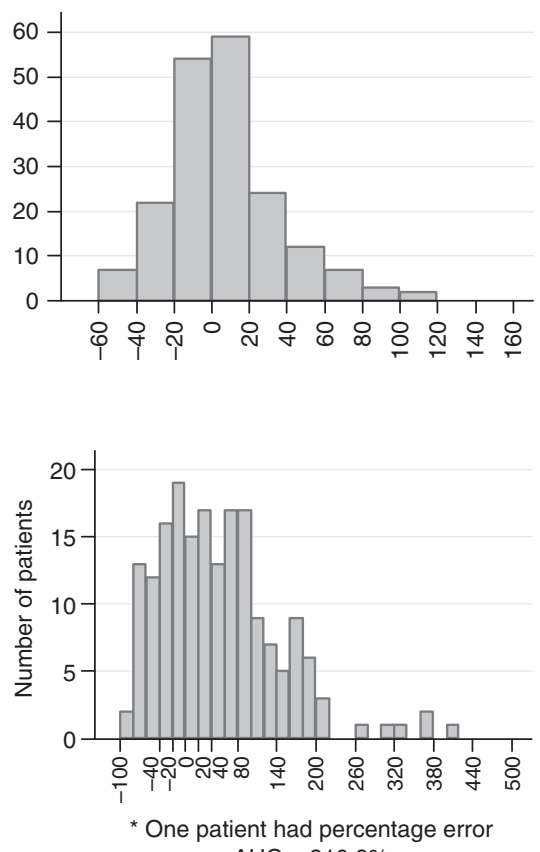

AUC $=810.9 \%$
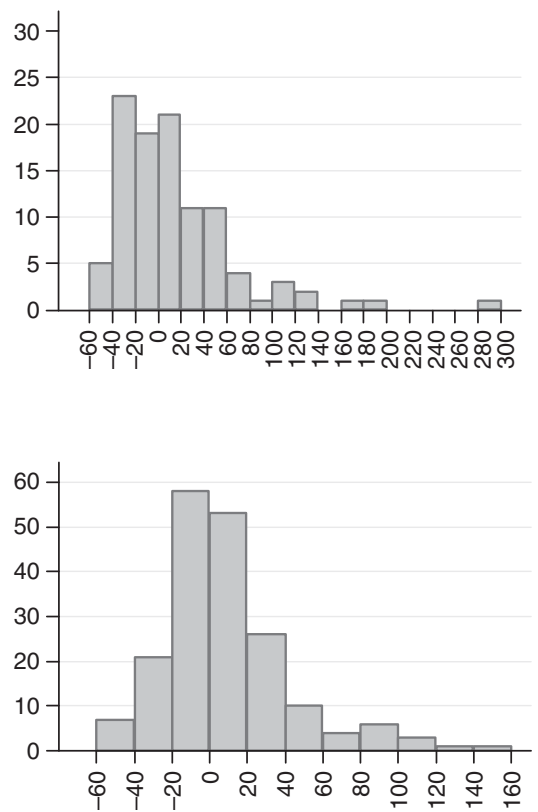

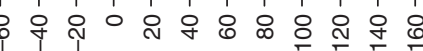

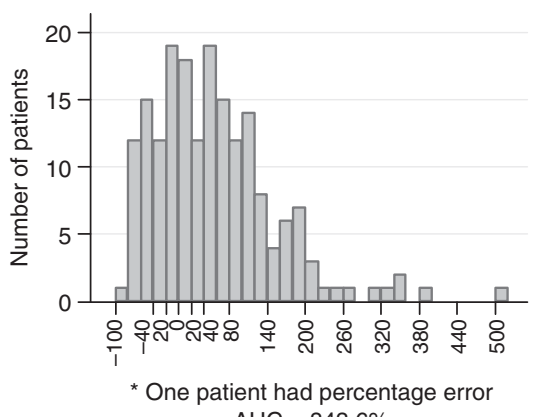

$\mathrm{AUC}=843.6 \%$

Figure 3 Frequency of percentage errors between individual AUC and target AUC using BSA dosing, dose banding, or fixed dose for doxorubicin, topotecan, and irinotecan (considering SN-38 concentrations).

the causes of interindividual PK variability, before administrating irinotecan (Deeken et al, 2008). There are at least two other drugs for which dose banding cannot be recommended from the present work. First, 5-flurouracil pharmacokinetics is non-linear due to saturable liver metabolism corresponding to DPD enzyme, such that even a small change in dose could be associated with large modifications in AUC. For example, considering a patient with typical values of $\mathrm{Km}$ and $\operatorname{Vmax}\left(5.57 \mathrm{mgl}^{-1}\right.$ and $51390 \mathrm{mgh}^{-1}$, respectively; Terret et al, 2000), an increase of $20 \%$ of 10 -min IV infusion of $500 \mathrm{mg} \mathrm{m}^{-2}$ would be associated with a $39 \%$ increase in AUC. The second drug is carboplatin, since for this agent, the dose is most often calculated from GFR to target a specified AUC (Jodrell, 1999). Dose banding in this case may be based on 'bands' of predicted carboplatin clearance. Additional work is needed to determine for which other drugs the proposed strategy might have relevance.

Analytical control of chemotherapy preparation is currently in a phase of generalisation. Some automated analytical robots are now available. This procedure represents a step forward in terms of increasing the safety of drug management from preparation to administration. One of its main limits is the delay that it creates between the physician's prescription and the administration of the drug to the patients. Dose banding would make it possible to anticipate chemotherapy preparation and analytical control without any delay for the patients.

In conclusion, the current evaluation of dose banding based on PK criteria confirms the feasibility of future implementation of this procedure in routine practice, and warrants its further evaluation in prospective clinical trials comparing the effect of BSA-dosing and dose-banding methods on toxicity and efficacy. In the present study, AUC was used as it represented the best available surrogate marker for the clinical consequences for the patient since only PK data were available, but in the prospective study we plan to set up, toxicity and efficacy will be assessed as well as plasma drug exposure. Although the patient's interest may not be evident at first, improvement of quality control and speed of availability of the chemotherapy on the day of the visit are two examples of benefit to the patient which could be derived from dose banding. Thus, in view of the important advantages of dose banding in terms of production time gain and reduction of wastage, it is of primary importance to evaluate whether it can be shown to be no worse than BSA dosing. This prospective study will concern some of the drugs evaluated and their inclusion criteria will include morphologic patients' characteristics corresponding to those of the patients in the present work. 


\section{REFERENCES}

Berhoune M, Aboudagga H, Jacob A, Grandhaye JP, Dayot C, Prognon P, Bonan B (2011) Effect of requiring advance approval of chemotherapy on number and cost of ungiven doses. Am J Health Syst Pharm 68(7): $557-558$

de Jong FA, Mathijssen RH, Xie R, Verweij J, Sparreboom A (2004) Flatfixed dosing of irinotecan: influence on pharmacokinetic and pharmacodynamic variability. Clin Cancer Res 10(12 Part 1): 4068-4071

de Jongh FE, Verweij J, Loos WJ, de WR, de Jonge MJ, Planting AS, Nooter K, Stoter G, Sparreboom A (2001) Body-surface area-based dosing does not increase accuracy of predicting cisplatin exposure. J Clin Oncol 19(17): 3733-3739

Deeken JF, Slack R, Marshall JL (2008) Irinotecan and uridine diphosphate glucuronosyltransferase $1 \mathrm{~A} 1$ pharmacogenetics: to test or not to test, that is the question. Cancer 113(7): 1502-1510

Gurney H (1996) Dose calculation of anticancer drugs: a review of the current practice and introduction of an alternative. J Clin Oncol 14(9): 2590-2611

Henningsson A, Marsh S, Loos WJ, Karlsson MO, Garsa A, Mross K, Mielke S, Vigano L, Locatelli A, Verweij J, Sparreboom A, McLeod HL (2005) Association of CYP2C8, CYP3A4, CYP3A5, and ABCB1 polymorphisms with the pharmacokinetics of paclitaxel. Clin Cancer Res 11(22): 8097-8104

Jodrell DI (1999) Formula-based dosing for carboplatin. Eur J Cancer 35(9): 1299-1301

Leger F, Loos WJ, Fourcade J, Bugat R, Goffinet M, Mathijssen RH, Verweij J, Sparreboom A, Chatelut E (2004) Factors affecting pharmacokinetic variability of oral topotecan: a population analysis. $B r$ J Cancer 90(2): 343-347
Mathijssen RH, de Jong FA, Loos WJ, van der Bol JM, Verweij J, Sparreboom A (2007) Flat-fixed dosing versus body surface area based dosing of anticancer drugs in adults: does it make a difference? Oncologist 12(8): 913-923

Mathijssen RH, van Alphen RJ, Verweij J, Loos WJ, Nooter K, Stoter G, Sparreboom A (2001) Clinical pharmacokinetics and metabolism of irinotecan (CPT-11). Clin Cancer Res 7(8): 2182-2194

Pinkel D (1958) The use of body surface area as a criterion of drug dosage in cancer chemotherapy. Cancer Res 18(7): 853-856

Plumridge RJ, Sewell GJ (2001) Dose-banding of cytotoxic drugs: a new concept in cancer chemotherapy. Am J Health Syst Pharm 58(18): 1760-1764

Pouliquen AL, Escalup L, Jourdan N, Cottu P, Faure P, MadelaineChambrin I (2011) Dose standardisation of anticancer drugs. Int J Clin Pharm 33(2): 221-228

Reilly JJ, Workman P (1993) Normalisation of anti-cancer drug dosage using body weight and surface area: is it worthwhile? A review of theoretical and practical considerations. Cancer Chemother Pharmacol 32(6): 411-418

Rudek MA, Sparreboom A, Garrett-Mayer ES, Armstrong DK, Wolff AC, Verweij J, Baker SD (2004) Factors affecting pharmacokinetic variability following doxorubicin and docetaxel-based therapy. Eur J Cancer 40(8): $1170-1178$

Sparreboom A (2005) BSA-based dosing and alternative approaches. Clin Adv Hematol Oncol 3(6): 448-450

Terret C, Erdociain E, Guimbaud R, Boisdron-Celle M, McLeod HL, FetyDeporte R, Lafont T, Gamelin E, Bugat R, Canal P, Chatelut E (2000) Dose and time dependencies of 5-fluorouracil pharmacokinetics. Clin Pharmacol Ther 68(3): 270-279

This work is published under the standard license to publish agreement. After 12 months the work will become freely available and the license terms will switch to a Creative Commons Attribution-NonCommercial-Share Alike 3.0 Unported License. 